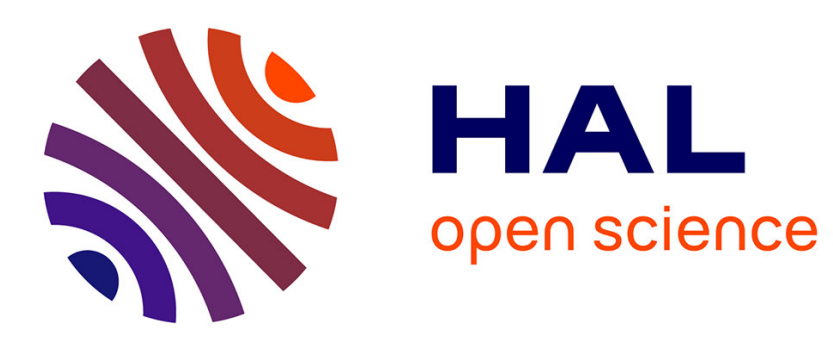

\title{
Sexe et usage de l'espace? L'influence de la répartition des rôles au sein du ménage
}

Odile Andan, Charles Raux

\section{To cite this version:}

Odile Andan, Charles Raux. Sexe et usage de l'espace? L'influence de la répartition des rôles au sein du ménage. Espace Populations Sociétés, 1986, 1, pp. 99-110. halshs-00324383

\section{HAL Id: halshs-00324383 \\ https://shs.hal.science/halshs-00324383}

Submitted on 22 Oct 2010

HAL is a multi-disciplinary open access archive for the deposit and dissemination of scientific research documents, whether they are published or not. The documents may come from teaching and research institutions in France or abroad, or from public or private research centers.
L'archive ouverte pluridisciplinaire HAL, est destinée au dépôt et à la diffusion de documents scientifiques de niveau recherche, publiés ou non, émanant des établissements d'enseignement et de recherche français ou étrangers, des laboratoires publics ou privés. 
Ce document vous est proposé avec l'aimable autorisation de l'éditeur scientifique et des auteurs, maîtres des droits. La présente version en PDF est sous le copyright de l’Université de Lille 1 (http://eps.revues.org/) - 1989. Ce document est protégé en vertu de la loi du droit d'auteur.

With the editors and the publisher of the copyright agreement. The present version in PDF is under the copyright of Université de Lille 1 (http://eps.revues.org/). 
Edité par l'Université des Sciences et Techniques de Lille - Flandres - Artois,

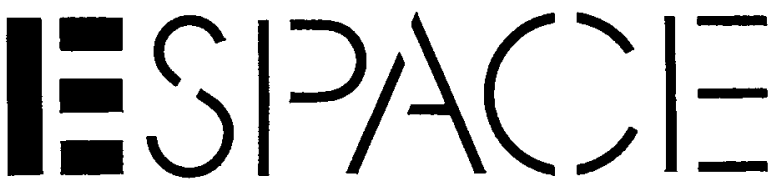

avec le concours des Universités de Picardie, Paris I, Liège,
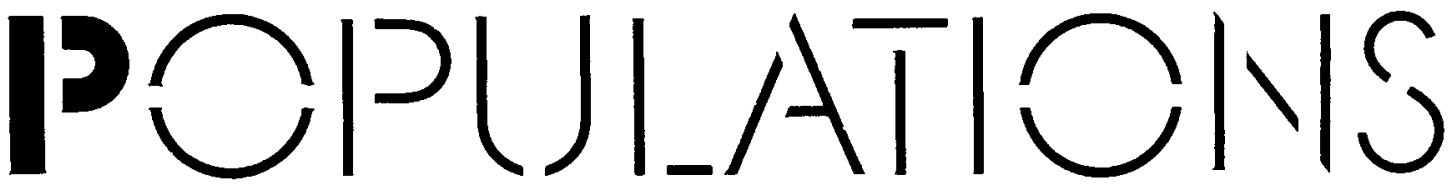

de l'Université libre de Bruxelles

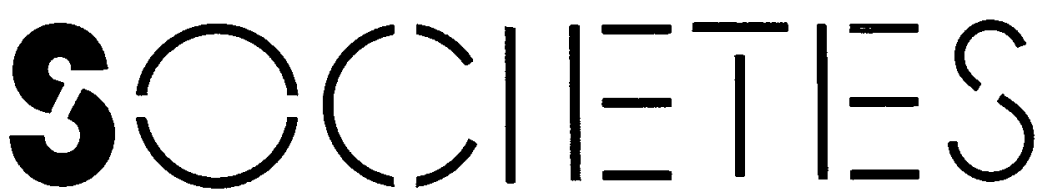

et de l'Université Catholique de Louvain

extrait extract 


\section{Sexe et usage de l'espace? L'influence de la répartition des rôles au sein du ménage}

\section{INTRODUCTION}

Sexe et usage de l'espace ? Existe-t-il un tel determinisme entre d'une part la différen ciation sexuelle entre hommes et femmes, d'autre part la manière dont l'espace est utilisé par les uns et les «unes»? Notre propos est de montrer que la différenciation sexuelle ne nous paraît pas intervenir de façon aussi directe et immuable sur les relations des individus à l'espace. Son action semble fortement médiatisée et modulé par la répartition des rôles au sein du ménage, qui évoluent sous l'effet de la conjoncture économique et sociale.

Pour ce faire, nous nous appuierons su deux exemples tirés de notre expérience. Il s'agit de deux enquêtes que nous avons menées sur les comportements de mobilite des individus, c'est-à-dire sur la manière dont ils se déplacent pour participer à des activités (travail, accompagnements d'enfants, achats, visites, etc.) localisées en des lieux précis de l'espace urbain. Selon cette optique particulière, nous avons en effet été amenés à rechercher comment les individus organisent leurs activités et quel sont les determinants de leur usage de 'espace pour pratiquer leurs activités.

L'organisation des activités des individus apparaît liée à la répartition des rôles au sein du ménage. Cette répartition est ellemême le résultat d'arbitrages entre les individus et leur entourage: entourage familial d'abord pour la répartition des charges familiales, social ensuite pour l'organisation spatio-temporelle des activités (travail école). Ces arbitrages font appel à des modèles que le ménage trouve dans la société et auxquels il se réfère pour engendrer ses pratiques quotidiennes. Ces modèles reposent sur des normes - rôles et valeurs - intériorisés par les individus et sont relatifs à la famille, au couple, aux rôles masculin et féminin.

C'est à la répartition des activités entre homme et femme au sein du ménage, qui dérive des modèles relatifs aux rôles, que nous nous intéresserons dans un premier temps. Dans un deuxième temps nous essaierons de voir si cette répartition donne leu chez les hommes et les femmes à des usages spécifiques de l'espace.

Toutefois la nature de nos données ne nous permet pas de traiter l'ensemble de ces rapports à l'espace relevant tant des mécanismes complexes de la perception que de ceux de la pratique. Nous ne pouvons ainsi abor- 
der ni la relation des individus à l'espace domestique, celui du logement, ni analyser les multiples aspects de la relation à l'espace du "dehors», extérieur au logement. Nous devons nous limiter à une partie de cet espace du dehors, l'espace « vécu»

\section{A. LES ENQUETES UTILISEES}

\section{L'enquête Paris-Marne}

Elles avait pour but d'étudier l'influence de la structure urbaine sur les comportements de mobilité des individus. Elle consistait à observer et comparer les comportements d'individus vivant successivement dans deux milieux urbains différents: Paris et Marne-la-Vallée, ville nouvelle en formation, située à $15 \mathrm{~km}$ à l'est de Paris (Andan et alii, 1980, 1982, 1984). L'enquête a été menée auprès de 71 ménages, sélectionnés selon cinq critères: âge, couple avec ou sans enfant, Parisiens devant s'installer à Marne, de nationalité française et futurs propriétaires à Marne-la-Vallée. Ces ménages appartiennent aux classes moyennes (cadres moyens et employés). Les deux membres du couple ont été interrogés. L'échantillon n'a pas pour but d'être représentatif, mais de saisir quelques mécanismes de la relation de la mobilité et de l'espace urbain.

L'enquête s'est déroulée en deux phases auprès des mêmes ménages: une première à Paris en 1980 avant le déménagement, une seconde après le déménagement à Marne en 1982. Aux changements apportés par le déménagement se sont ajoutés pour certains ménages dans cet intervalle, d'autres changements (naissance d'un enfant, cessation de l'activité professionnelle pour les femmes). Deux outils

\section{B. ROLES ET PRATIQUES DES ACTIVITES AU SEIN DU MENAGE}

Les rôles qu'assume chaque individu relèvent tant du domaine de la production que de celui de la reproduction ou des relations sociales: ils génèrent une diversité d'activités dont certaines sont réalisées dans l'espace privé du domicile et d'autres à l'extérieur de cet espace privé. C'est à ces activités «d'extérieur» que nous limiterons d'enquête ont été utilisés: un questionnaire concernant les caractéristiques des individus et du ménage et un carnet de bord relevant, sur une période d'une semaine, tous les déplacements effectués par les individus.

\section{L'enquête Lyon}

L'enquête Lyon a été réalisée (Raux, 1986) sur l'agglomération lyonnaise ( 1 million d'habitants) en 1984. Elle avait pour but de montrer les conséquences de la participation croissante des femmes à la force de travail, sur l'organisation des activités et des déplacements dans leurs ménages. Elle visait plus particulièrement à montrer les différences de comportement selon trois critères: l'âge des enfants, le statut matrimonial de la famille (couple ou famille mono-parentale) et l'activité ou non de l'épouse. Cette enquête s'inscrivait dans une recherche internationale plus large (Rosenbloom, 1983).

L'enquête a été réalisée auprès de 150 ménages répartis en six groupes de 25 ménages en moyenne, selon ces trois criteres. L'échantillon reflète globalement le profil socio-économique de l'agglomération lyonnaise. Il n'est bien sûr aucunement représentatif, les chiffres présentés pou cette enquête n'ayant d'intérêt que dans le cadre d'une analyse comparative interne entre tel ou tel sous-groupe de l'échantillon

notre propos: l'activité professionnelle et parmi les activités d'entretien de la famille, les accompagnements d'enfants et les achats.

1. L'activité professionnelle des femmes Actuellement en règle générale, les ressour ces financières du ménage sont encore prio- ritairement assurées par le travail de l'homme malgré le développement de l'activité professionnelle des femmes. Si l'activité professionnelle de l'homme paraît aller de soi, il n'en est pas encore de même pour la femme. Son insertion dans le monde de la production dépend en partie d'un arbitrage au sein du couple.

L'enquête Lyon a essayé d'identifier les termes de cet arbitrage: les réponses montrent qu'assez peu de femmes déclarent ne vouloir prendre de travail sous aucun prétexte:

$4 \%$ chez les mères de jeunes enfants, $16 \%$ chez les mères d'enfants de plus de six ans. La principale raison puur prendre un travail serait une obligation de nature économique: soit par nécessité financière, soit en cas de chômage du conjoint : cela est vrai pour les deux tiers des mères de famille quel que soit l'âge des enfants. Les autres raisons sont liées à la nécessité de suivre les enfants, qui empêche la prise d'un travail, pour les mères de jeunes enfants $(39 \%)$, et à la qualité du travail pour les mères d'enfants de plus de six ans ( horaires souples» ou «travail intéressant»). Pour ce dernier groupe de femmes inactives, rester à la maison paraît plus un choix qu'une obligation liée aux enfants. Pour la plupart d'entre elles, en effet, le travail ne présente guère d'intérêt pour lui-même : il est avant tout un gagne-pain.

Les femmes ont en outre un comportement moins stable que les hommes par rapport à l'emploi, même si elles interrompent de moins en moins souvent leur activité professionnelle et si elles la reprennent plus tôt et plus fréquemment (Huet, Schmitz, 1984). L'enquête Paris-Marne montre qu'elles prennent ou abandonnent le travail selon les circonstances: entre 1980 (Paris) et 1982 (Marne), $19 \%$ des femmes avaient changé de statut d'emploi, c'est-àdire soit abandonné, soit pris un travail. Cette instabilité est également confirmée par l'enquête Lyon qui a analysé les réactions éventuelles à de fortes contraintes économiques sur le mode de garde des enfants, lorsque les parents travaillent. L'effet testé était celui d'un doublement des coûts actuels du mode de garde, y compris toutes les autres options possibles de garde des enfants. Un certain nombre de parents ne se sentaient pas concernés: les maris dont la femme ne travaillait pas et les parents d'adolescents ou d'enfants scolarisés. La comparaison entre femmes et hommes à l'intérieur de chaque groupe de couples de deux actifs montre que les réactions varient en fonction de l'âge des enfants. La différence est très nette entre hommes et femmes avec jeunes enfants: $64 \%$ des hommes déclarent qu'en cas d'un tel doublement des coûts des modes de garde, ils ne changeraient rien, contre $30 \%$ pour les femmes; la principale réaction des femmes serait d'arrêter de travailler $(30 \%)$. Les hommes et femmes avec enfant de plus de six ans se sentent moins concernés et déclarent à égalité (50 et $54 \%$ ) que ce doublement des coûts n'aurait aucun effet.

Enfin, la comparaison entre les mères de jeunes enfants révèle des différences de comportement entre celles qui vivent en couple et celles qui vivent seules. Les mères seules sont plus nombreuses que celles en couple $(41 \%$ contre $30 \%)$ à déclarer que ce doublement des coûts n'aurait aucun effet. De plus aucune d'elles n'arrêterait de travailler: la plupart chercheraient un autre arrangement $(32 \%)$ ou déclarent qu'elles se trouveraient dans l'impossibilité d'assurer la garde de leurs enfants $(23 \%)$ : c'est donc plus de la moitié $(55 \%)$ d'entre elles qui se trouveraient fortement contraintes puisque ne pouvant arrêter de travailler De même les mères d'enfants de plus de six ans sont dans une même proportion (50\%) à déclarer que ce doublement des coûts n'aurait aucun effet mais là encore aucune des mères seules n'envisage d'arrêter de travailler.

Cet exemple montre que «l'instabilité » des femmes vis-à-vis de l'activité professionnelle varie selon leurs situations. Il se confirme que le statut familial est le facteur qui influence le plus le comportement d'activité professionnelle des femmes: les femmes seules, quel que soit l'âge des enfants, ne peuvent s'arrêter de travailler, alors que les femmes vivant en couple en ont la possibilité. Le second facteur qui intervient est l'âge des enfants: l'instabilité apparaît d'autant plus marquée que l'enfant est jeune.

2. Les charges familiales

Les tâches d'entretien domestique et d'édu- 
cation des enfants constituent un ensemble diversifié dans leurs rapports avec la mobilité : l'entretien du foyer, la préparation des repas, les soins aux enfants sont des travaux intérieurs, alors que les achats, les accompagnements d'enfants, les démarches diverses provoquent des déplacements. Tout aussi variées soient-elles, elles sont culturellement reliées par le fait qu'elles sont principalement féminines.

Cependant, comme nous venons de le voir deux paramètres modulent pour les femmes le poids des charges familiales à assumer : la présence ou l'absence de mari d'une part, le fait d'avoir ou non une activité professionnelle d'autre part. Inactive la femme supporte tout le poids des charges familiales, active et mariée elle doit parfois interrompre son activité professionnelle pour résoudre de trop fortes contraintes, active et seule elle doit s'adapter à ces contraintes sans pouvoir abandonner le travail.

a) Les accompagnements des enfants L'éducation des enfants entraîne des servitudes diverses, tant au dehors qu'audedans du foyer. Mais le problème de mobilité qu'elle induisent se pose dans des termes différents selon l'âge des enfants.

Tableau 1 : Pourcentage des personnes qui combinent des déplacements domicile-travail avec d'autres motifs

\begin{tabular}{|l|c|c|c|c|c|c|}
\hline & \multicolumn{3}{|c|}{ Aller } & \multicolumn{3}{|c|}{ Retour } \\
\hline & T.motifs & Enfants & Autres & T. motifs & Enfants & Autres \\
\hline Total & $44 \%$ & $35 \%$ & $9 \%$ & $69 \%$ & $32 \%$ & $37 \%$ \\
Femmes & $24 \%$ & $13 \%$ & $11 \%$ & $36 \%$ & $11 \%$ & $25 \%$ \\
Hommes & & & & & & \\
$\begin{array}{l}\text { Couples de 2 actifs, } \\
\text { jeunes enfants }\end{array}$ & $39 \%$ & $35 \%$ & $4 \%$ & $65 \%$ & $34 \%$ & $31 \%$ \\
$\begin{array}{l}\text { Femmes } \\
\text { Hommes }\end{array}$ & $38 \%$ & $28 \%$ & $10 \%$ & $43 \%$ & $29 \%$ & $14 \%$ \\
$\begin{array}{l}\text { Couples de 2 actifs, } \\
\text { enfants de plus de 6 ans }\end{array}$ & $23 \%$ & $12 \%$ & $11 \%$ & $62 \%$ & $19 \%$ & $43 \%$ \\
$\begin{array}{l}\text { Femmes } \\
\text { Hommes }\end{array}$ & $23 \%$ & $12 \%$ & $11 \%$ & $31 \%$ & $12 \%$ & $19 \%$ \\
$\begin{array}{l}\text { Mères seules } \\
\text { de jeunes enfants } \\
\text { d'enfants de plus de 6 ans }\end{array}$ & $86 \%$ & $72 \%$ & $14 \%$ & $91 \%$ & $55 \%$ & $36 \%$ \\
$\begin{array}{l}\text { Couple un actif, père } \\
\text { de jeunes enfants } \\
\text { d'enfants de plus de 6 ans }\end{array}$ & $22 \%$ & $40 \%$ & $8 \%$ & $65 \%$ & $26 \%$ & $39 \%$ \\
\hline
\end{tabular}

Source: Enquête Lyon.

Par ailleurs, mêrne si la manière dont les parents éduquent leurs enfants s'inscrit toujours dans un projet de reproduction sociale, les pratiques et les perspectives varient avec les groupes.

Dans la période du premier âge, la mère a comme alternative de rester à la maison ou de se déplacer pour confier l'enfant à une nourrice ou une crèche. La solution d'arrêter ou de différer son activité professionnelle est souvent adoptée comme étant normale.

Les stratégies de la reproduction présentes dans l'idée qu'il faut sortir les petits enfants, s'affirment et se diversifient lorsque les enfants grandissent. Les déplacements pour aller à l'école reposent sur un fonds commun, structuré par l'âge des enfants et la succession des établissements qu'ils fréquentent. Les problèmes les plus aigus se posent lorsque la femme travaille et que la famille veut élargir le cadre de l'éducation des enfants. L'adaptation peut résulter soit de l'abandon du travail par la mère - ou d'une reprise retardée au-delà du premier âge -, soit d'une aide extérieure lorsque les moyens du couple le permettent, soit enfin d'une autonomisation précoce du déplacement des enfants.
Les activités des enfants sont marquées par les fixités spatiales (localisations de ces activités) et temporelles (horaires), qui s'imposent à leurs parents: cette fixité spatio temporelle implique pour les parents des contraintes particulières d'usage du temps et de l'espace.

Qui accompagne les enfants lorsqu'ils on des activités extérieures au domicile: école, sport ou loisirs? Ces accompagnements ont été analysés dans l'enquête Lyon par le biais de l'organisation des déplacements domicile-travail des hommes et femmes actifs.

Ces déplacements domicile-travail son organisés bien différemment selon la situation familiale de l'individu (Tableau 1). Les femmes combinent en général plus souven que les hommes leurs déplacements domicile-travail avec d'autres motifs, le principal étant l'accompagnement des enfants à l'aller et les courses au retour: c'est vrai à l'aller et plus encore au retour. Ces comportements moyens masquent des effets différenciés selon la situation matrimoniale ou d'activité: hommes et femmes des ménages à deux actifs partagent quasiment à égalité les accompagnements à l'aller, quel que soit l'âge des enfants; par contre les hommes s'impliquent moins que leurs épouses lorsqu'elles ne travaillent pas. Les mères seules de jeunes enfants sont $86 \%$ à l'aller et $91 \%$ au retour à effectue de telles combinaisons, témoignant d'une situation de forte contrainte.

Les différences de comportement d'accompagnement s'observent donc, d'une part entre hommes et femmes des couples de deux actifs et femmes inactives, d'autre part entre les parents d'enfants de moins de 6 ans et les autres. Si les hommes apparaissent moins contraints que les femmes par les accompagnements des enfants, ils partagent cependant plus volontiers ces accompagnements quand leur femme travaille et quand les enfants sont jeunes.

Cette répartition des accompagnements ne peut-elle être perturbée par l'impact des formes spatiales urbaines (localisation des activités, offre de transport)? C'est à cette question que l'enquête Paris-Marne permet d'apporter des éléments de réponse.

Contrairement à ce que l'on attend, malgré l'alourdissement des contraintes
Tableau 2: Evolution de la répartition des accompagnements de membres du ménage dans les familles avec enfants, entre 1980 (Paris) et 1982 (Marne)

\begin{tabular}{|l|r|r|}
\hline $\begin{array}{l}\text { Accompagnements } \\
\text { dans la semaine }\end{array}$ & 1980 & 1982 \\
\hline L'homme uniquement & $10 \%$ & $9 \%$ \\
La femme uniquement & $20 \%$ & $24 \%$ \\
L'homme ct la femme & $45 \%$ & $41 \%$ \\
Aucun d'entre eux & $25 \%$ & $26 \%$ \\
\hline Total & $100 \%$ & $100 \%$ \\
\hline
\end{tabular}

Source: Enquête Paris-Marne.

entraîné par les nouvelles naissances et le déménagement vers un milieu mal équipé, il n'y a pas de transfert de ces activités d'accompagnements des femmes vers les hommes. Il y aurait même une légère tendance inverse, même si globalement on peut considérer qu'il n'y a aucun changement dans la répartition des accompagnements sur l'échantillon observe.

b) Les achats

Parmi les achats, il y a lieu de distinguer ceux réalisés pour l'alimentation et l'entretien domestique usuel, de ceux pour l'entretien et l'équipement de la personne et de la maison.

Les premiers donnent lieu, selon une pratique traditionnelle, à des déplacements relativement réglés et fréquents. Ils sont surtout assurés par les femmes et principalement par celles qui ne travaillent pas, même si les hommes peuvent y participer dans certaines circonstances. Cela résulte encore de la division générale des rôles qui donne aux hommes le travail à l'extérieur et aux femmes le travail domestique. Dans le cas des femmes sans profession, la prise en charge de ces courses revêt un caractère d'évidence. Il est entendu que l'homme n'a pas le temps ou ne va pas ressortir en rentrant du travail. Toutefois avec l'essor des grandes surfaces, l'approvisionnement hebdomadaire massif fait en couple tend aujourd'hui à se développer et à remplacer la pratique classique des courses quotidiennes.

Les achats d'entretien et d'équipement génèrent des déplacements beaucoup moins réguliers. Bien que visant des biens domes- 
tiques, ils entrent dans la rationalité économique de la sphère ménagère et à ce titre impliquent davantage les hommes. C'est qu'il ne s'agit plus seulement d'acquérir les matériaux nécessaires à la cuisine et au ménage, mais d'équiper la famille et le foyer. L'importance des investissements, le prolongement des achats par l'aménagement et le bricolage, introduisent l'homme dans la gestion des achats.

Quelques chiffres issus de l'enquête ParisMarne attestent bien ce type de répartition $67 \%$ des femmes actives et $64 \%$ des fem mes inactives font des achats courants contre $35 \%$ des hommes seulement. Par contre, si l'on prend l'ensemble des démarches d'achat (courants, groupés et occasionnels), la participation des hommes augmente $(68 \%)$ mais celle des femmes encore davantage $(90 \%)$ !

En conséquence, les modes de vie des femmes actives sont plus fortement marqués par les activités domestiques (achats et accompagnements) que ceux de leurs conjoints. Pour les deux tiers d'entre elles, leur emploi du temps des jours ouvrables se réduit à la pratique d'activités contraintes (travail, charges familiales et domestiques), alors que la moitié des hommes seulemen sont dans la même situation. Malgré les obligations liées à l'agrandissement de la famille entre 1980 et 1982, un quart d'entre eux contre $12 \%$ des femmes se soustraien à des charges, consacrant la semaine à l'activité professionnelle, à laquelle ils ajoutent exceptionnellement quelques activités de loisirs (Tableau 3).

En outre, même s'ils ont le même schéma d'activité, les hommes et les femmes répar tissent différemment leur déplacements entre les activités: chez les femmes, les acti-

\section{LA RELATION A L'ESPACE}

De quelle manière les différences que nous avons tenté de dégager entre hommes et femmes dans l'exercice de leurs activités, se réflètent-elles dans leurs rapports respectifs à l'espace?

L'espace fréquenté pour ces différentes activités s'organise-t-il par rapport au domicile différemment pour les hommes et

(1) Dans l'enquête Paris-Marne, cet espace est mesur par l'ensemble des points de l'espace, définis par coor-
Tableau 3: Répartition des individus selon la combinaison des activités qu'ils ont pratiquées les jours ouvrés

\begin{tabular}{|l|r|r|}
\hline Types d'activités & Hommes & Femmes \\
\hline Travail, activités & & \\
domestiques, loisirs & $24 \%$ & $25 \%$ \\
Travail, activités & & \\
domestiques & $51 \%$ & $63 \%$ \\
Travail, loisirs & $3 \%$ & $5 \%$ \\
Travail & $22 \%$ & $7 \%$ \\
\hline Total & $\mathbf{1 0 0} \%$ & $\mathbf{1 0 0} \%$ \\
\hline
\end{tabular}

Source: Enquête Paris-Marne, 1982

vités domestiques occupent toujours une part plus importante dans leur emploi du temps, tandis que les loisirs en occupent une moindre, par rapport aux hommes. Cependant, avec les changements liés au déménagement et à l'accroissement de la famille, les hommes procèdent à quelques ajustements de leur emploi du temps (léger accroissement du poids des activités domestiques, baisse de la part des loisirs), alors que les femmes présentent une certaine stabilité, quel que soit leur schéma d'activité. Certes les hommes participent davantage aux charges domestiques mais les femmes en gardent la responsabilité. Redistribution des tâches à l'intérieur du couple? Pas vraiment! Dans la mesure où les femmes peuvent plus difficilement assumer ces charges, les hommes sembleraient plutôt intervenir pour soulager les femmes d'une partie de celles-ci, plutôt que pour les remplacer. Sans profession, la femme doit s'adapter à la mobilité des autres, conjoint et enfants: la reproduction domestique constitue l'essentiel de ses activités et justifie la sédentarité. Active, elle doit intégrer sa propre mobilité de travail à la vie domestique.

les femmes? Les rythmes de fréquentation traduisent-ils les contraintes temporelles de leurs schémas d'activités respectifs?

\section{Organisation de l'espace vécu par} rapport au domicile

L'espace vécu (1) des femmes se distingue$\mathrm{t}$-il sensiblement de celui des hommes?

données géographiques, visités par les individus durant la période d'observation de l'enquête.
Dans l'enquête Paris-Marne, comme on peut s'y attendre, il existerait bien une relation entre la fréquentation de l'espace urbain et le sexe et l'activité professionnelle des individus. Mais le clivage s'opère essentiellement entre les femmes sans profession et hommes et femmes actifs. L'espace fréquenté par les femmes inactives est en 1982 pour les deux tiers, centré sur la ville nouvelle, leur lieu de résidence, tandis que pour les hommes et les femmes actifs, il est orienté vers Paris et la banlieue $(77 \%$ pour les hommes et $70 \%$ pour les femmes).

Si, dans notre exemple, l'espace d'activité des actifs - hommes et femmes - apparaît selon l'expression d'A. Haumont «déterritorialisé », c'est en raison de la séparation des lieux de travail et de domicile. Cette séparation est d'origine non pas géographique mais sociale, résultant d'un processus social profond qui a séparé le domicile des salariés urbains de leur lieu de travail. Bien qu'ils habitent Marne-la-Vallée, $11 \%$ des hommes et $16 \%$ des femmes seulement ont un emploi à Marne-la-Vallée ou dans les proches environs alors que $69 \%$ des hommes et $71 \%$ des femmes travaillent encore à Paris intra-muros. Cet éloignement entre la résidence et le travail n'apparaît guère être voulu, mais résulte le plus souvent des difficultés du marché de l'emploi et de celui du logement. En effet, pas plus ces ménages que ceux de l'enquête Lyon ne paraissent avoir la maîtrise de cet écart domicile-travail. Parmi ces derniers, $70 \%$ déclarent qu'il n'y a aucune relation entre le choix de leur lieu de domicile et celui du lieu de travail.

L'accessibilité temporelle et spatiale du travail est donc dépendante de l'articulation spatiale entre lieu de résidence et d'emploi à l'intérieur d'un même milieu urbain, des conditions de desserte ainsi que de la plus ou moins bonne adaptation des horaires de travail aux autres activités des individus. Cette accessibilité joue sur les modes de vie des individus un rôle important, dans la mesure où elle concerne l'activité la plus longue et la moins flexible dans le temps

(2) Les lieux de résidence, travail, etc., sont des sec(2) Les liex de residence, travall, ecc., sont des secfonction de la place qu'ils occupent dans la vie quolidienne des individus. En raison de problemes tech- et dans l'espace, autour de laquelle s'ordonne l'ensemble des activités de la journée ou de la semaine.

L'activité professionnelle influence dans une certaine mesure la localisation des achats des actifs comme nous le constatons dans l'enquête Paris-Marne (Tableau 4).

Tableau 4: Localisation des achats selon le sexe et l'activité professionnelle des individus $(\mathrm{en} \%)(\mathrm{H}=$ hommes, $\mathrm{FA}=$ femmes actives, FIA = femmes inactives)

\begin{tabular}{|l|c|c|c|c|c|c|}
\hline Lieux (2) & \multicolumn{3}{|c|}{1980} & \multicolumn{3}{c|}{1982} \\
\hline & H & FA & FIA & H & FA & FIA \\
\hline & $\%$ & $\%$ & $\%$ & $\%$ & $\%$ & $\%$ \\
\hline Résidence & 55 & 62 & 74 & 18 & 25 & 38 \\
Travail & 10 & 5 & - & 5 & 8 & - \\
Trajet D-T & - & - & - & 9 & 16 & - \\
Autres lieux & 35 & 33 & 26 & 68 & 51 & 62 \\
\hline Total & $\mathbf{1 0 0}$ & $\mathbf{1 0 0}$ & $\mathbf{1 0 0}$ & $\mathbf{1 0 0}$ & $\mathbf{1 0 0}$ & $\mathbf{1 0 0}$ \\
\hline
\end{tabular}

Source: Enquête Paris-Marne.

En 1980, où les personnes de l'échantillon habitent Paris, on observe plus de différences entre les inactifs et les actifs, qu'entre hommes et femmes. Les femmes sans profession concentrent $74 \%$ de leurs achats à proximité de leur domicile, sensiblement plus que les femmes actives $(62 \%)$ et les hommes actifs $(55 \%)$. L'importance que les femmes sans profession accordent à leur quartier de résidence est bien sûr à mettre en relation avec le type d'achats qu'elles y font, achats alimentaires qui sont les plus fréquents.

Cependant les qualités de l'environnement peuvent amener à modifier les règles de ces pratiques d'achat. En effet en 1982, les femmes comme les hommes résidant dans des quartiers peu ou non équipés en commerces courants, ne font désormais qu'une faible part de leurs achats près du domicile. La différence entre actifs et sans profession s'observe toujours, mais fait nouveau, les femmes actives se distinguent aussi des hommes. Elles font sensiblement

niques de mesure, les lieux de résidence et de iravail hiques de mesure, les lieux de résidence et de iravail domicile et du ravail et facilographiques proches du 
plus que ceux-ci leurs achats dans les espaces qui leur sont facilement accessibles: lieu de résidence, lieu de travail, trajet domiciletravail $(49 \%$ contre $32 \%)$.

En outre, ce changement de localisation des achats s'accompagne d'une certaine remise en cause de la pratique traditionnelle des courses fréquentes, tant chez les femmes actives et inactives que chez les hommes. En 1982, les grandes surfaces attirent une plus forte proportion d'entre eux : $40 \%$ des hommes au lieu de $15 \%$ en $1980,40 \%$ des femmes actives au lieu de $14 \%$ et $34 \%$ des inactives au lieu de 7\%. Cette modification des comportements est liée à la fois à l'inadaptation des horaires des commerces aux contraintes des actifs et à la faiblesse des équipements commerciaux de leurs quartiers d'habitation. Tout les incite à regrouper davantage leurs achats en une seule sortie dans les grandes surfaces, bien représentées à Marne-la-Vallée. Toutefois la fréquentation de ces grandes surfaces entraîne pour les usagers l'élargissement de leur espace de consommation et le recours à la voiture.

\section{L'interdépendance entre la vie hors} domicile et la vie au domicile

L'exercice d'une activité professionnelle paraît donc être le principal facteur de la discrimination entre les espaces d'activité des hommes et des femmes. Le clivage s'opère davantage entre femmes sans profession et hommes et femmes actifs que seulement entre hommes et femmes. En effet, les espaces d'activité des femmes actives sont, comme ceux des hommes, essentiellement organisés autour de l'activité la plus prégnante, le travail. L'inégal partage des responsabilités d'entretien domestique et d'éducation ne transparaît guère. Il est vrai aussi qu'une partie seulement de cellesci donnent lieu à sortie hors domicile. Si les femmes actives ne limitent point par rapport aux hommes le champ spatial de leurs déplacements, leur vie à l'extérieur du domicile serait par contre empreinte de la lourdeur de leurs charges.

Le temps que les femmes passent hors du domicile semblerait effectivement plus compté que celui des hommes (Tableau 5). Il existerait bien sûr pour tous, une limite qu'ils ne dépassent pas: aux dates d'en-
Tableau 5: Répartition des actifs selon le pourcentage de temps qu'ils ont passé hors du domicile les jours ouvrés

\begin{tabular}{|l|r|r|r|r|}
\hline & \multicolumn{2}{|c|}{ Hommes } & \multicolumn{2}{c|}{ Femmes } \\
\hline$\%$ de temps & 1980 & 1982 & 1980 & 1982 \\
\hline Plus de $50 \%$ & $26 \%$ & $19 \%$ & $11 \%$ & $5 \%$ \\
50 à $40 \%$ & $65 \%$ & $64 \%$ & $81 \%$ & $84 \%$ \\
Moins de $40 \%$ & $9 \%$ & $17 \%$ & $8 \%$ & $11 \%$ \\
\hline Total & $100 \%$ & $100 \%$ & $100 \%$ & $100 \%$ \\
\hline
\end{tabular}

Source: Enquête Paris-Marne 1982

quête, $80 \%$ des actifs au moins, hommes et femmes, ne sont pas plus de 12 heures hors de chez eux. Toutefois, les femmes sont plus nombreuses que les hommes à rester moins de 12 heures dehors. Elles ont par ailleurs un comportement assez uniforme: en 1980 et en 1982 , plus de $80 \%$ d'entre elles (65\% des hommes) passent 40 à $50 \%$ de leur temps hors du domicile; les hommes ont des comportements plus diversifiés: 26 à $19 \%$ sont plus de 12 heures dehors ( 11 à $5 \%$ des femmes), 9 à $17 \%$ moins de 10 heures dehors $(8$ à $9 \%$ des femmes). Pourquoi ces différences? Volonté de gagner du temps ou plus grande difficulté à s'adapter aux nouvelles conditions de vie? Nos données ne permettent pas d'y répondre.

D'autres types de données permettent de constater que, plus que chez les hommes, la sortie du domicile est effectivement influencée chez les femmes par les obligations familiales et domestiques. Ce phénomène de dépendance se manifeste dans plusieurs domaines: l'organisation des aspects temporels du travail, l'ordonnancement des sorties.

D'après l'enquête Lyon, l'interaction entre organisation du travail et organisation des activités des enfants dans le temps ne concerne pratiquement que les femmes ( $38 \%$ à $65 \%$ selon les groupes définis par les situations familiales). Le plus souvent d'ailleurs, il s'agit d'un arrangement des horaires de travail en fonction des activités des enfants plutôt que le contraire. Seules les mères de jeunes enfants font mention du travail à temps partiel ( 20 à $25 \%)$; les femmes concernées font en général les horaires variables ou ne travaillent pas du tout ou partiellement le mercredi. Par ailleurs les mères seules de jeunes enfants se déclarent le plus obligées d'arranger les activités de leurs enfants en fonction de leur travail tandis que les femmes vivant en couple, à l'inverse ont dû le plus souvent organiser leur travail en fonction de leurs enfants; la présence d'un autre adulte dans le ménage ne semble donc pas, au contraire, alléger

les charges qui pèsent sur les mères actives.

Elles sont en conséquence plus vulnérables à tout changement affectant les conditions temporelles du travail: durée du trajet domicile-travail, horaires. Cette vulnérabilité a pu être appréciée dans l'enquête Lyon, à travers les réactions déclarées à d'éventuelles modifications de l'offre en transport. L'ensemble des actifs sont plus sensibles à la durée qu'au coût des déplacements domicile-travail, les femmes pour la plupart ( 50 à $74 \%$ ) plus que les hommes (19 à $48 \%$ ). Les femmes les plus gênées pa cette contrainte temporelle sont les mères de jeunes enfants vivant en couple $(74 \%)$ tandis que leur conjoints le seraient le moins (19\%). Les femmes des couples avec enfants de plus de 6 ans seraient moins nombreuses à réagir $(50 \%$ seulement $)$.

La structuration des sorties des femmes actives paraît aussi être conditionnée par les pressions de leur vie à la maison. Dans l'enquête Paris-Marne, bien que vivant successivement dans deux contextes urbains différents, les femmes actives sont aussi

nombreuses à limiter le nombre de leurs sorties à une seule, celle du travail : $59 \%$ à Paris et $61 \%$ à Marne-la-Vallée. Eviter d'autres allers-retours à partir du domicile peut être considéré comme une façon de gagner du temps.

Si l'on compare les comportements de sortie des individus vivant successivement à Paris et Marne-la-Vallée, on constate que la stabilité de comportement n'est pas du tout la même chez les hommes et chez les femmes. Alors que chez les hommes il n'y a guère de relation entre ce qu'ils ont fait en 1980 et 1982 on observe chez les femmes une plus grande similitude (Tableau 6). Les hommes sont une minorité à n'avoir pas fait le déplacement hors travail aux deux dates et il y a indépendance complète de leurs comportements. Les femmes en majorité n'en ont pas fait en 1980 et les trois quarts d'entre elles sont restées dans la même catégorie en 1982. On retrouve ici un résultat souvent observé ailleurs: les femmes ont un comportement plus réglé que les hommes, conséquence probablement à la fois des contraintes matérielles et contraintes de rôles, les hommes pouvant se permettre plus de fantaisie.

En conséquence, plus volontiers que les hommes, les femmes mettent à profit la sortie liee au travail pour pratiquer d'autres activités: $74 \%$ en 1980 et $69 \%$ en 1982 pour les femmes, 58 et $60 \%$ pour les hommes.

Tableau 6: Répartition des individus selon la présence ou l'absence de sortie hors travail (en nombre)

\begin{tabular}{|l|c|c|c|l|c|c|c|}
\hline \multicolumn{3}{|c|}{ Hommes } & \multicolumn{4}{c|}{ Femmes } \\
En 1982 1982 \\
\hline En 1980 & Non & Oui & Total & En 1980 & Non & Oui & Total \\
\hline Non & 9 & 15 & 24 & Non & 15 & 5 & 20 \\
Oui & 10 & 23 & 33 & Oui & 5 & 9 & 14 \\
\hline Total & $\mathbf{1 9}$ & $\mathbf{3 8}$ & $\mathbf{5 7}$ & Total & $\mathbf{2 0}$ & $\mathbf{1 4}$ & $\mathbf{3 4}$ \\
\hline
\end{tabular}

Source: Enquête Paris-Marne 1982

\section{CONCLUSION}

Sexe et usage de l'espace? La relation estelle si évidente? Nos observations n'ont aucune prétention à la généralisation, mais malgré leur limites, elles montrent que cette relation est complexe.

Elle apparaît prioritairement conditionnée par la répartition des rôles au sein du ménage. Pour les actifs l'espace d'activité 
est principalement organisé autour de l'axe domicile-travail, tandis que pour les sansprofession il est marqué par les activités domestiques centrées pour la plupart autour du domicile. Dans la mesure où aujourd'hui les femmes participent de plus en plus nombreuses au monde de la production, les différences deviennent plus sensibles entre actifs (hommes et femmes) et femmes inactives, que simplement entre hommes et femmes.

Cependant les charges familiales interviennent toujours pour maintenir aussi un clivage entre hommes et femmes actives. Malgré le développement de l'activité professionnelle des femmes, le modèle relatif aux rôles féminins et masculins évolue peu. Si changement il y a, il apparaît plutôt au niveau de l'exercice de ces diverses activités qu'à celui des responsabilités à assumer. La responsabilité de l'entretien domestique et d'éducation des enfants revient encore aux femmes.

Ces charges familiales agissent indirectement sur les espaces d'activité des femmes, en leur imposant des contraintes de temps. Ces contraintes limitent ainsi leur liberte d'action en dehors du domicile, en les obligeant à gagner du temps de diverses façons: aller dans les lieux les plus rapidement accessibles, coordonner au mieux leurs activités dans l'espace et le temps, grouper les activités en un minimum de sorties pour éviter les aller-retour au domicile. L'influence d'autres facteurs peut relativi- ser les observations que nous avons faites sur la répartition des rôles et la relation à l'espace:

la position du ménage dans le cycle de vie: femmes et hommes partagent plus volontiers les tâches familiales quand les enfants sont jeunes, même si les femmes en gardent la responsabilité;

- la qualité de l'environnement urbain: il peut rendre impossible ou du moins difficile tel ou tel comportement et en permettre d'autres. L'espace de consommation des femmes inactives et actives par exemple, a dû changer entre Paris et Marne, à cause du faible équipement de leur lieux de résidence en commerces traditionnels; - les ressources du ménage: elles dépendent des caractéristiques du couple. Cette recherche, tout en faisant apparaître le jeu des contraintes imposées aux modes de vie, met en évidence les ressources dont disposent les individus pour élaborer des stratégies. Ainsi quand les charges familiales sont pressantes, les femmes vivant en couple ont par rapport aux femmes seules le "choix»" de rester à la maison, dans la mesure où le travail du conjoint garantit une certaine entrée d'argent. Selon les moyens financiers dont dispose le couple, la femme peut recourir à une aide extérieure pour garder ses enfants. Ces stratégies sont donc un moyen de gérer, voire négocier les contraintes dues aux normes imposées: ces contraintes peuvent être poussées ou accentuées ou au contraire contrecarrées.

\section{REFERENCES BIBLIOGRAPHIQUES}

ANDAN (O.), COUTRAS (J.), CURRAT (C.), MATALON (B), 1980. Mobilité et espace urbain. Etude longitudinale des comportements de mobilite en fonction d'un changement de résidence (Paris, Laboratoire Analyse de l'Espace, rapport préléminaire pour l'Institut de Recherche des Transports, $40 \mathrm{p}$. et annexes)

ANDAN (O.), ASKEVIS (F.), CURRAT (C.), MATALON (B) POITEVINEAU (J) REICHMAN (S.), 1982. Mobilité et espace urbain. Etude longitudinale des comportements de mobilite en fonction d'un dinale des comportements de mobilite en fonction d'un changement de residence. Avant demenagement intermédiaire pour EPA-Marne, 150 p. et anexor)

ANDAN (O.), ASKEVIS (F.), CURRAT (C.), MATALON (B), POITEVINEAU (J.), REICHMAN (S.), SALOMON (I.), 1984. Mobilité et espace urbain.
HUET (M.), SCHMITZ (N.), 1984. L'évolution des emplois (in Données Sociales 1984, INSEE, Paris)

RAUX (C.), 1986. Relations entre travail, charges familiales et transport en France (Lyon, Laboratoire d'Economie des Transports, note de travail, 79 p. et annexes).

RAIX (C.), ROSENBLOOM (S.), 1986. Employment, childcare and travel behavior: France, the
Netherlands, the United States (in Behavioural research for transport policy, VNU Science Press, pp. 363-380).

ROSENBLOOM (S.), 1983. The transportation planning implications of the growing work force participation of women with children in industrial countries France, the Netherlands and the United States (The University of Texas at Austin, working paper).
Etude longitudinale des comportements de mobilité en fonction d'un changement de résidence (Paris, Laboratoire Analyse de l'Espace, rapport final pour la Mission de la Recherche du Ministère des Trans. ports, $200 \mathrm{p}$. et annexes)

AVEROUS (B.), MATALON (B.), GODARD (X.), ORFEUIL (I P.) 1979. Recherche sur la mobilite des personnes en zone urbaine Motorisation et usage individuel de la voilure (Paris, rapport de recherche IRT no 38 , tome II).

HAUMONT (A.), LAMBERTI (A.), VIETDAPAULE (N.), WINTERSDORFF (F.), VIETDEPaUté (N.). WINTERSDRFF (F.), 1977. La Mobilité et espaces urbains, Paris, mode de vie (in 
Adresse: Espace-Populations-Sociétés,

Université des Sciences et Techniques de Lille - Flandres - Artois U.F.R. de Géographie,

59655 Villeneuve d'Ascq Cedex, France

tél. 20436552 